\title{
Lower and upper bounds for $H$-eigenvalues of even order real symmetric tensors
}

\author{
Hongwei Jin* \\ M. Rajesh Kannan ${ }^{\dagger}$ \\ Minru Bai $\ddagger$
}

September 14, 2018

\begin{abstract}
In this article, we define new classes of tensors called double $\bar{B}$-tensors, quasi-double $\bar{B}$-tensors and establish some of their properties. Using these properties, we construct new regions viz., double $\bar{B}$-intervals and quasi-double $\bar{B}$-intervals, which contain all the $H$-eigenvalues of real even order symmetric tensors. We prove that the double $\bar{B}$-intervals is contained in the quasi-double $\bar{B}$-intervals and quasi-double $\bar{B}$-intervals provide supplement information on the Brauer-type eigenvalues inclusion set of tensors. These are analogous to the double $\bar{B}$-intervals of matrices established by J. M. Peña [On an alternative to Gerschgorin circles and ovals of Cassini, Numer. Math. 95 (2003), no. 2, 337-345.]
\end{abstract}

AMS Subject Classification(2010): 15A18, 15A69, 65F15.

Keywords: double $\bar{B}$-tensors, quasi-double $\bar{B}$-tensors, double $\bar{B}$-intervals, quasi-double $\bar{B}$-intervals, Brauer-type eigenvalues inclusion theorem, $H$-eigenvalues, $Z$-tensors.

\section{Introduction}

A tensor can be regarded as a higher-order generalization of a matrix, which takes the form

$$
\mathcal{A}=\left(a_{i_{1} \ldots i_{m}}\right), \quad a_{i_{1} \ldots i_{m}} \in \mathbb{R}, \quad i_{j} \in[n]:=\{1, \ldots, n\}, \quad j \in[m]
$$

Such a multidimensional array is called an $m$-order $n$-dimensional real tensor and the set of all $m$-order $n$-dimensional real tensors is denoted by $\mathcal{T}\left(\mathbb{R}^{n}, m\right)$. A tensor $\mathcal{A}$ is called symmetric if its entries $a_{i_{1} \ldots i_{m}}$ are invariant under any permutation of their indices $\left\{i_{1}, \ldots, i_{m}\right\}$.

\footnotetext{
${ }^{*}$ College of Mathematics and Econometrics, Hunan University, 410082, Changsha, P.R. China. Email: hw-jin@hotmail.com

${ }^{\dagger}$ Department of Mathematics, Technion - Israel Institute of Technology, Haifa 32000, Israel. Email: rajeshkannan1.m@gmail.com

${ }^{\ddagger}$ College of Mathematics and Econometrics, Hunan University, 410082, Changsha, P.R. China. E-mail: minru-bai@163.com
} 
Let $\mathbb{R}^{n}\left(\mathbb{C}^{n}\right)$ denote the $n$-dimensional real (complex) vector space. Vectors are denoted by lower case letters $(x, y, \ldots)$, matrices by upper case letters $(A, B, \ldots)$ and tensors by calligraphic upper case letters $(\mathcal{A}, \mathcal{B}, \ldots)$. The $i^{t h}$ entry of a vector $x$ is denoted by $x_{i}$, the $(i, j)^{t h}$ entry of a matrix $A$ is denoted by $a_{i j}$ and the $\left(i_{1}, \ldots, i_{m}\right)^{t h}$ entry of a tensor $\mathcal{A}$ is denoted by $a_{i_{1} \ldots i_{m}}$.

Given a vector $x=\left(x_{1}, \ldots, x_{n}\right)^{T} \in \mathbb{C}^{n}$, we define $\mathcal{A} x^{m-1}$ to be a vector in $\mathbb{C}^{n}$ whose $i$ th coordinate is

$$
\left(\mathcal{A} x^{m-1}\right)_{i}=\sum_{i_{2}, \ldots, i_{m}=1}^{n} a_{i i_{2} \ldots i_{m}} x_{i_{2}} \cdots x_{i_{m}} .
$$

For $x \in \mathbb{C}^{n}$ and a natural number $k$, the vector $x^{[k]}$ is the Hadamard power of $x$, i.e. $x_{i}^{[k]}=x_{i}^{k}$ for all $i$.

Definition 1.1. [8] Let $\mathcal{A} \in \mathcal{T}\left(\mathbb{R}^{n}, m\right)$. If there is a nonzero vector $x \in \mathbb{C}^{n}$ and a number $\lambda \in \mathbb{C}$ such that

$$
\mathcal{A} x^{m-1}=\lambda x^{[m-1]}
$$

, then $\lambda$ is called an eigenvalue of the tensor $\mathcal{A}$ and $x$ an eigenvector of $\mathcal{A}$ associated with $\lambda$. Furthermore, we say $\lambda$ is an $H$-eigenvalue with the corresponding $H$-eigenvector of $\mathcal{A}$ if they are real.

A symmetric tensor $\mathcal{A} \in \mathcal{T}\left(\mathbb{R}^{n}, m\right)$ is said to be positive semidefinite if for any vector $x \in \mathbb{R}^{n}, \mathcal{A} x^{m}:=\sum_{i_{1}, \ldots, i_{m}=1}^{n} a_{i_{1} \ldots i_{m}} x_{i_{1}} \cdots x_{i_{m}} \geq 0 ; \mathcal{A}$ is positive definite if for any nonzero vector $x \in \mathbb{R}^{n}, \mathcal{A} x^{m}>0$. From the definition it is clear that, if $m$ is odd, then there is no nontrivial positive semidefinite tensor.

Positive definite homogenous polynomials and positive semidefinite polynomials (nonnegative polynomials) are important in the field of dynamical systems, optimization, etc. With each homogenous polynomial we can associate a symmetric tensor. Checking the positive (semi)definiteness of a homogenous polynomial is equivalent to checking the positive (semi)definiteness of the symmetric tensor associated with it. For details about the applications we refer to [6], [8] and the references therein.

Qi characterized the positive definite and positive semidefinite tensors in terms of their $H$-eigenvalues.

Theorem 1.1. [8, Theorem 5] Let $\mathcal{A}$ be an m-order $n$-dimensional symmetric tensor such that $m$ is an even integer. Then $\mathcal{A}$ is positive definite (positive semidefinite) if and only if all its $H$-eigenvalues are positive (nonnegative).

Thus, from the above theorem, the location of the $H$-eigenvalues of an even order symmetric tensor is useful in checking the positive definiteness (positive semidefiniteness) of tensors. The main purpose of this article is to give upper and lower bounds for the $H$-eigenvalues of even order symmetric tensors. Next we recall a couple of known results in this direction.

For each $i \in[n]$, denote

$$
r_{i}(\mathcal{A})=\sum\left\{\left|a_{i i_{2} \ldots i_{m}}\right|: i_{j} \in[n], j=2, \ldots, m,\left(i_{2}, \ldots, i_{m}\right) \neq(i, \ldots, i)\right\} .
$$


Qi et al. [8, 9] asserted that the eigenvalues of a tensor $\mathcal{A}$ have a similar statement as the Gerschgorin circle of the eigenvalues of matrices. One can rewrite the result for $H$-eigenvalues of a real tensor as follows.

Theorem 1.2. [9, Theorem 2] Let $\mathcal{A}=\left(a_{i_{1} \ldots i_{m}}\right) \in \mathcal{T}\left(\mathbb{R}^{n}, m\right)$ and $\lambda$ be an $H$-eigenvalue of $\mathcal{A}$. Then,

$$
\lambda \in \Gamma(\mathcal{A})=\bigcup_{i=1}^{n} \Gamma_{i}(\mathcal{A})
$$

where $\Gamma_{i}(\mathcal{A})=\left[a_{i \ldots i}-r_{i}(\mathcal{A}), a_{i \ldots i}+r_{i}(\mathcal{A})\right]$.

We call the interval $\Gamma(\mathcal{A})$ in (4) the Gerschgorin eigenvalues inclusion set of tensors. Li et al. established a Brauer-type eigenvalues inclusion set for an arbitrary complex tensor [4, Theorem 2.1] and showed that the Brauer-type eigenvalues inclusion set is contained in the Gerschgorin eigenvalues inclusion set [4, Theorem 2.3]. We can restate the Brauer-type eigenvalues inclusion theorem for $H$-eigenvalues of a real tensor as follows:

Theorem 1.3. 4, Theorem 2.1] Let $\mathcal{A}=\left(a_{i_{1} \ldots i_{m}}\right) \in \mathcal{T}\left(\mathbb{R}^{n}, m\right)$ and $\lambda$ be an $H$-eigenvalue of $\mathcal{A}$. Then,

$$
\lambda \in \Omega(\mathcal{A})=\bigcup_{\substack{i, j=1 \\ i \neq j}}^{n} \Omega_{i j}(\mathcal{A})
$$

where

$$
\Omega_{i j}(\mathcal{A})=\left\{z \in \mathbb{C}:\left|z-a_{i \ldots i}\right|\left(\left|z-a_{j \ldots j}\right|-r_{j}^{i}(\mathcal{A})\right) \leq r_{i}(\mathcal{A})\left|a_{j i \ldots i}\right|\right\},
$$

and

$$
r_{j}^{i}(\mathcal{A})=r_{j}(\mathcal{A})-\left|a_{j i \ldots i}\right|=\sum_{\substack{j_{2}, \ldots, j_{m}=1 \\\left(j_{2}, \ldots, j_{m}\right) \neq(i, \ldots, i) \\\left(j_{2}, \ldots, j_{m}\right) \neq(j, \ldots, j)}}^{n}\left|a_{j j_{2} \ldots j_{m}}\right| .
$$

In this article, in Section 2, we collect some definitions, known results and correct minor mistakes in couple of results proved in [3]. In Section 3, we define two new classes tensors viz., double $\bar{B}$-tensors, quasi-double $\bar{B}$-tensors and establish their properties. Using this properties, in Section 4, we construct two new regions called double $\bar{B}$-intervals and quasidouble $\bar{B}$-intervals, containing all the $H$-eigenvalues of even order symmetric tensors. These regions have a nature similar to the Brauer-type eigenvalues inclusion set $\Omega(\mathcal{A})$ stated in (5). We prove that quasi-double $\bar{B}$-intervals is smaller than double $\bar{B}$-intervals and there is no inclusion relation between quasi-double $\bar{B}$-intervals and the Brauer-type eigenvalues inclusion set. Hence, it is nature to construct an intervals $\Upsilon(\mathcal{A})$, narrower than both quasidouble $\bar{B}$-intervals and Brauer-type eigenvalues inclusion set $\Omega(\mathcal{A})$, by intersecting the two. These comparison results are done in Section 5. In Section 6, we draw some concluding remarks. 


\section{Notation, Definitions and Preliminary results}

The purpose of this section is twofold - to collect known definitions and results; and to correct minor mistakes in couple of results in [3].

The $m$-order $n$-dimensional identity tensor, denoted by $\mathcal{I}$, is the tensor with entries

$$
a_{i_{1} \ldots i_{m}}= \begin{cases}1, & \text { if } i_{1}=\cdots=i_{m} \\ 0, & \text { otherwise }\end{cases}
$$

Chen et al. [1] defined the following $k$ th row tensor dealing with some problems of circulant tensors.

Definition 2.1. [1] Let $\mathcal{A}=\left(a_{i_{1} \ldots i_{m}}\right) \in \mathcal{T}\left(\mathbb{R}^{n}, m\right)$. Then, $\mathcal{A}_{k}=\left(a_{i_{1} \ldots i_{m-1}}^{(k)}\right) \in \mathcal{T}\left(\mathbb{R}^{n}, m-1\right)$ is called the $k$ th row tensor if $a_{i_{1} \ldots i_{m-1}}^{(k)}=a_{k i_{1} \ldots i_{m-1}}$, where $k, i_{1}, \ldots, i_{m-1} \in[n]$.

Let $\operatorname{sign}(x)$ be sign function, that is,

$$
\operatorname{sign}(x)= \begin{cases}1, & x>0 \\ 0, & x=0 \\ -1, & x<0\end{cases}
$$

For a tensor $\mathcal{A}=\left(a_{i_{1} \ldots i_{m}}\right) \in \mathcal{T}\left(\mathbb{R}^{n}, m\right)$ and $i \in[n]$, define

$$
\begin{gathered}
\beta_{i}(\mathcal{A})=\max \left\{0, a_{i i_{2} \ldots i_{m}}:\left(i_{2}, \ldots, i_{m}\right) \neq(i, \ldots, i)\right\}, \\
\gamma_{i}(\mathcal{A})=\min \left\{0, a_{i i_{2} \ldots i_{m}}:\left(i_{2}, \ldots, i_{m}\right) \neq(i, \ldots, i)\right\}, \\
\Delta_{i}(\mathcal{A})=\sum\left\{\left(\beta_{i}(\mathcal{A})-a_{i i_{2} \ldots i_{m}}\right):\left(i_{2}, \ldots, i_{m}\right) \neq(i, \ldots, i)\right\}, \\
\Delta_{j}^{i}(\mathcal{A})=\sum\left\{\left(\beta_{j}(\mathcal{A})-a_{j j_{2} \ldots j_{m}}\right):\left(j_{2}, \ldots, j_{m}\right) \neq(i, \ldots, i),\left(j_{2}, \ldots, j_{m}\right) \neq(j, \ldots, j)\right\}, \\
\Theta_{i}(\mathcal{A})=\sum\left\{\left(a_{i i_{2} \ldots i_{m}}-\gamma_{i}(\mathcal{A})\right):\left(i_{2}, \ldots, i_{m}\right) \neq(i, \ldots, i)\right\}
\end{gathered}
$$

and

$$
\Theta_{j}^{i}(\mathcal{A})=\sum\left\{\left(a_{j j_{2} \ldots j_{m}}-\gamma_{j}(\mathcal{A})\right):\left(j_{2}, \ldots, j_{m}\right) \neq(i, \ldots, i),\left(j_{2}, \ldots, j_{m}\right) \neq(j, \ldots, j)\right\}
$$

Next we recall the definitions of double $B$-tensor and quasi-double $B$-tensor defined by Li et al. in [3].

Definition 2.2. Let $\mathcal{A}=\left(a_{i_{1} \ldots i_{m}}\right) \in \mathcal{T}\left(\mathbb{R}^{n}, m\right)$ with $a_{i_{\ldots i}}>\beta_{i}(\mathcal{A})$ for all $i$. Then $\mathcal{A}$ is said to be a double $B$-tensor if :

(a) for any $i \in\{1, \ldots, n\}, a_{i \ldots i}-\beta_{i}(\mathcal{A}) \geq \Delta_{i}(\mathcal{A})$,

(b) for all $i, j \in\{1, \ldots, n\}, i \neq j,\left(a_{i \ldots i}-\beta_{i}(\mathcal{A})\right)\left(a_{j \ldots j}-\beta_{j}(\mathcal{A})\right)>\Delta_{i}(\mathcal{A}) \Delta_{j}(\mathcal{A})$. 
Note that Chen et al. 2] also gave a definition of double $B$-tensor, which is different from the above one. A double $B$-tensor defined in [2] need not satisfy the condition $(a)$ of definition 2.2. If we drop condition $(a)$ from definition 2.2 , then even order symmetric double $B$-tensors need not be positive definite 3 . Positive definiteness of double $B$-tensors plays a crucial role in locating the $H$-eigenvalues of even order symmetric tensors. So, in this paper, we follow the definition of double $B$-tensor as in [3].

Definition 2.3. [3] Let $\mathcal{A}=\left(a_{i_{1} \ldots i_{m}}\right) \in \mathcal{T}\left(\mathbb{R}^{n}, m\right)$ with $a_{i \ldots i}>\beta_{i}(\mathcal{A})$ for all $i$. Then $\mathcal{A}$ is said to be a quasi-double $B$-tensor if :

$$
\left(a_{i \ldots i}-\beta_{i}(\mathcal{A})\right)\left(a_{j \ldots j}-\beta_{j}(\mathcal{A})-\Delta_{j}^{i}(\mathcal{A})\right)>\left(\beta_{i}(\mathcal{A})-a_{j i \ldots i}\right) \Delta_{j}(\mathcal{A}) .
$$

Next we recall some definitions.

Definition 2.4. [3] Let $\mathcal{A}$ be an $m$-order $n$-dimensional tensor. Then $\mathcal{A}$ is called a doubly strictly diagonally dominant tensor (DSDD) if:

(a) $\left|a_{i \ldots i}\right|\left|a_{j \ldots j}\right|>r_{i}(\mathcal{A}) r_{j}(\mathcal{A})$, for all $i, j, i \neq j$

(b) when $m>2,\left|a_{i \ldots i}\right| \geq r_{i}(\mathcal{A})$ for $i \in\{1, \ldots, n\}$.

Definition 2.5. [3] Let $\mathcal{A}$ be an $m$-order $n$-dimensional tensor. Then $\mathcal{A}$ is called a quasidoubly strictly diagonally dominant tensor ( $Q-D S D D)$ if:

$$
\left|a_{i \ldots i}\right|\left(\left|a_{j \ldots j}\right|-r_{j}^{i}(\mathcal{A})\right)>r_{i}(\mathcal{A})\left|a_{j i \ldots i}\right|, \text { for all } i, j, i \neq j \text {. }
$$

A tensor $\mathcal{A}$ is called a $Z$-tensor if there exists a tensor $\mathcal{D}$ with nonnegative entries and a real number $s$ such that $\mathcal{A}=s \mathcal{I}-\mathcal{D}$.

Li et al. [3] proved that the following important result using the fact that an even order symmetric double $B$-tensor (quasi-double $B$-tensor) can be decomposed into the sum of a doubly (quasi-doubly) strictly diagonally dominant symmetric $Z$-tensor and several positive multiples of partially all one tensors.

Theorem 2.1. 3] The following statements are true:

(a) All the $H$-eigenvalues of an even order symmetric double B-tensor are positive,

(b) All the $H$-eigenvalues of an even order symmetric quasi-double B-tensor are positive.

The following results are proved in [3]. We observed that these results contain minor mistakes. We give a counter example to these results and prove the correct versions (Theorem 3.1, Theorem 2.4).

Theorem 2.2. [3, Proposition 5] Let $\mathcal{A}$ be an m-order $n$-dimensional Z-tensor. Then:

(a) $\mathcal{A}$ is a double B-tensor if and only if $\mathcal{A}$ is a DSDD tensor.

(b) $\mathcal{A}$ is a quasi-double $B$-tensor if and only if $\mathcal{A}$ is a $Q$-DSDD tensor. 
Theorem 2.3. [3, Theorem 4] Let $\mathcal{A}$ be an m-order n-dimensional real symmetric tensor such that $m$ is even. If $\mathcal{A}$ is either a DSDD tensor or a $Q-D S D D$ tensor, then $\mathcal{A}$ is positive definite.

The following example shows that Theorem 2.2 and 2.3 are not true. Consider the $Z$ matrix $A=\left(\begin{array}{cc}-1 & -\frac{1}{2} \\ -\frac{1}{2} & -1\end{array}\right)$. Then $A$ is both DSDD and Q-DSDD-matrix. But, it is neither a double $B$-matrix nor a quasi-double $B$-matrix. Also it is easy to see that $A$ is not positive definite.

We will give a correct version of Theorem 2.2 in Section 3 . Now we give a correct version of Theorem 2.3 as the follows.

Theorem 2.4. Let $\mathcal{A}$ be an $m$-order $n$-dimensional real symmetric tensor such that $m$ is even and $a_{i \ldots i}>0$ for all $i \in[n]$.

(1) If $\mathcal{A}$ is a DSDD tensor, then $\mathcal{A}$ is positive definite;

(2) If $\mathcal{A}$ is a $Q-D S D D$ tensor, then $\mathcal{A}$ is positive definite;

Proof. The conclusion (1) follows directly from [5, Theorem 11]. If $\mathcal{A}$ is a Q-DSDD tensor, then it is easy to see that there exist an index $i \in[n]$ such that $\left|a_{i \ldots i}\right|>r_{i}(\mathcal{A})$. Now the conclusion (2) follows directly from [5, Theorem 13].

\section{Double $\bar{B}$-tensors and quasi-double $\bar{B}$-tensors}

In this section, first we introduce the notion of double $\bar{B}$-tensor and quasi-double $\bar{B}$-tensor.

Definition 3.1. Let $\mathcal{A}=\left(a_{i_{1} \ldots i_{m}}\right) \in \mathcal{T}\left(\mathbb{R}^{n}, m\right)$ be a tensor with the $k$ th row tensor $\mathcal{A}_{k}$, $k \in[n]$ and $\overline{\mathcal{A}}=\left(\bar{a}_{i_{1} \ldots i_{m}}\right) \in \mathcal{T}\left(\mathbb{R}^{n}, m\right)$ be a tensor with the $k$ th row tensor $\operatorname{sign}\left(a_{k \ldots k}\right) \mathcal{A}_{k}$, $k \in[n]$. Then,

(a) $\mathcal{A}$ is called a double $\bar{B}$-tensor if $\overline{\mathcal{A}}$ is a double $B$-tensor,

(b) $\mathcal{A}$ is called a quasi-double $\bar{B}$-tensor if $\overline{\mathcal{A}}$ is a quasi-double $B$-tensor.

Note that, double $\bar{B}$-tensors discussed in this paper is different from double $\bar{B}$-tensors discussed in [2] because of the different definitions of double $B$-tensor.

Let $\mathcal{A}$ be a double $B$-tensor. Since $a_{i \ldots i}>\beta_{i}(\mathcal{A}) \geq 0$ for each $i \in[n]$, it is easy to see that $\overline{\mathcal{A}}$ is a double $B$-tensor, which means $\mathcal{A}$ is a double $\bar{B}$-tensor. Hence,

$$
\{\text { double } B \text {-tensors }\} \subset\{\text { double } \bar{B} \text {-tensors }\} \text {. }
$$

Similarly, we have

\{quasi-double $B$-tensors $\} \subset$ quasi-double $\bar{B}$-tensors $\}$.

Define

$$
\delta_{i_{1} \ldots i_{m}}= \begin{cases}1, & \text { if } i_{1}=\cdots=i_{m} \\ 0, & \text { otherwise }\end{cases}
$$

Next we prove a correct version of Theorem 2.2. 
Theorem 3.1. Let $\mathcal{A}$ be an m-order $n$-dimensional tensor such that $\overline{\mathcal{A}}$ is a $Z$-tensor. Then

(a) $\mathcal{A}$ is a double $\bar{B}$-tensor if and only if $\mathcal{A}$ is a DSDD tensor.

(b) $\mathcal{A}$ is a quasi-double $\bar{B}$-tensor if and only if $\mathcal{A}$ is a $Q$-DSDD tensor.

Proof. (a) Let $\mathcal{B}=\overline{\mathcal{A}}$. Since $\mathcal{B}$ is a $Z$-tensor, we have $\beta_{i}(\mathcal{B})=0$ and $r_{i}(\mathcal{B})=\Delta_{i}(\mathcal{B})=$ $\sum_{\delta_{i i_{2} \ldots i_{m}}=0}-b_{i i_{2} \ldots i_{m}}$ for all $i$. Hence,

$$
\left|b_{i \ldots i}\right|\left|b_{j \ldots j}\right|>r_{i}(\mathcal{B}) r_{j}(\mathcal{B})
$$

if and only if

$$
\left(b_{i \ldots i}-\beta_{i}(\mathcal{B})\right)\left(b_{j \ldots j}-\beta_{j}(\mathcal{B})\right)>\Delta_{i}(\mathcal{B}) \Delta_{j}(\mathcal{B}) .
$$

Also $b_{i \ldots i}-\beta_{i}(\mathcal{B}) \geq \Delta_{i}(\mathcal{B})$ if and only if $\left|a_{i \ldots i}\right| \geq r_{i}(\mathcal{A})$. Thus $\mathcal{A}$ is a double $\bar{B}$-tensor if and only if $\mathcal{A}$ is a DSDD tensor.

(b)Now, $\Delta_{j}^{i}(\mathcal{B})=\sum_{\substack{\delta_{i j_{2} \ldots j_{m}}=0 \\ \delta_{j j_{2} \ldots j_{m}}=0}}\left(\beta_{j}(\mathcal{B})-b_{j j_{2} \ldots j_{m}}\right)=r_{j}^{i}(\mathcal{B})$. Hence

$$
b_{i \ldots i}\left(b_{j \ldots j}-r_{j}^{i}(\mathcal{B})\right)>r_{i}(\mathcal{B})\left|b_{j i \ldots i}\right|
$$

if and only if

$$
\left(b_{i \ldots i}-\beta_{i}(\mathcal{B})\right)\left(b_{j \ldots j}-\beta_{j}(\mathcal{B})-\Delta_{j}^{i}(\mathcal{B})\right)>\left(\beta_{j}(\mathcal{B})-b_{j i \ldots i}\right) \Delta_{i}(\mathcal{B}) .
$$

Thus $\mathcal{A}$ is a quasi double $\bar{B}$-tensor if and only if $\mathcal{A}$ is a Q-DSDD tensor.

Let us recall the general product of two $n$-dimensional tensors defined by Shao in [10].

Definition 3.2. 10] Let $\mathcal{A}=\left(a_{i_{1} \ldots i_{m}}\right) \in \mathcal{T}\left(\mathbb{C}^{n}, m\right)$ and $\mathcal{B}=\left(b_{i_{1} \ldots i_{k}}\right) \in \mathcal{T}\left(\mathbb{C}^{n}, k\right)$. Define the product $\mathcal{A B}$ to be the following tensor $\mathcal{C}$ of order $(m-1)(k-1)+1$ and dimension $n$ :

$$
c_{i \alpha_{1} \ldots \alpha_{m-1}}=\sum_{i_{2}, \ldots, i_{m}=1}^{n} a_{i i_{2} \ldots i_{m}} b_{i_{2} \alpha_{1}} \cdots b_{i_{m} \alpha_{m-1}}, \quad\left(i \in[n], \alpha_{1}, \ldots, \alpha_{m-1} \in[n]^{k-1}\right) .
$$

By the definition of double $\bar{B}$-tensor and quasi-double $\bar{B}$-tensor, it is easy to prove the following.

Theorem 3.2. Let $\mathcal{A}=\left(a_{i_{1} \ldots i_{m}}\right) \in \mathcal{T}\left(\mathbb{R}^{n}, m\right)$. Then

(a) $\mathcal{A}$ is a double $\bar{B}$-tensor if and only if there exists an $n \times n$ diagonal matrix $D$ whose diagonal elements belong to the set $\{1,-1\}$ and a double $B$-tensor $\mathcal{B} \in \mathcal{T}\left(\mathbb{R}^{n}, m\right)$ such that $\mathcal{A}=D \mathcal{B}$,

(b) $\mathcal{A}$ is a quasi-double $\bar{B}$-tensor if and only if there exists an $n \times n$ diagonal matrix $D$ whose diagonal elements belong to the set $\{1,-1\}$ and a quasi-double $B$-tensor $\mathcal{B} \in \mathcal{T}\left(\mathbb{R}^{n}, m\right)$ such that $\mathcal{A}=D \mathcal{B}$. 
Definition 3.3. Let $\mathcal{A}=\left(a_{i_{1} \ldots i_{m}}\right)$ be an $m$-order $n$-dimensional tensor and $\alpha \subseteq\{1, \ldots, n\}$ with $|\alpha|=r$. A principal subtensor $\mathcal{A}[\alpha]$ of the tensor $\mathcal{A}$ with index set $\alpha$ is an $m$-order $r$-dimensional subtensor of $\mathcal{A}$ consisting of $r^{m}$ elements defined as follows:

$$
\mathcal{A}[\alpha]=\left(a_{i_{1} \ldots i_{m}}\right) \text {, where } i_{1}, \ldots, i_{m} \in \alpha
$$

Theorem 3.3. Let $\mathcal{A}$ be an $m$-order $n$-dimensional tensor.

(a) If $\mathcal{A}$ is a double $\bar{B}$-tensor, then $\mathcal{A}[\alpha]$ is a double $\bar{B}$-tensor for all $\alpha \subseteq\{1, \ldots, n\}$.

(b) If $\mathcal{A}$ is a quasi-double $\bar{B}$-tensor, then $\mathcal{A}[\alpha]$ is a quasi-double $\bar{B}$-tensor for all $\alpha \subseteq$ $\{1, \ldots, n\}$.

Proof. (a) Let $\mathcal{B}=\overline{\mathcal{A}}, \alpha \subseteq\{1, \ldots, n\}$ and $i \in \alpha$. Since $b_{i \ldots i}>\beta_{i}(\mathcal{B})$, we have $b_{i \ldots i}>\beta_{i}(\mathcal{B}[\alpha])$. Also, $b_{i \ldots i}-\beta_{i}(\mathcal{B}) \geq \Delta_{i}(\mathcal{B})$ and $\Delta_{i}(\mathcal{B}) \geq \Delta_{i}(\mathcal{B}[\alpha])$. Thus $b_{i \ldots i}-\beta_{i}(\mathcal{B}[\alpha]) \geq \Delta_{i}(\mathcal{B}[\alpha])$. Now,

$$
\begin{aligned}
\left(b_{i \ldots i}-\beta_{i}(\mathcal{B}[\alpha])\right)\left(b_{j \ldots j}-\beta_{j}(\mathcal{B}[\alpha])\right) & \geq\left(b_{i \ldots i}-\beta_{i}(\mathcal{B})\right)\left(b_{j \ldots j}-\beta_{j}(\mathcal{B})\right) \\
& >\Delta_{i}(\mathcal{B}) \Delta_{j}(\mathcal{B}) \\
& \geq \Delta_{i}(\mathcal{B}[\alpha]) \Delta_{j}(\mathcal{B}[\alpha]) .
\end{aligned}
$$

Hence $\mathcal{A}[\alpha]$ is double $\bar{B}$-tensor.

(b) Let $i, j \in \alpha$ and $i \neq j$. We have $\Delta_{j}^{i}(\mathcal{B}) \geq \Delta_{j}^{i}(\mathcal{B}[\alpha])$. Now,

$$
\begin{aligned}
\left(b_{i \ldots i}-\beta_{i}(\mathcal{B}[\alpha])\right)\left(b_{j \ldots j}-\beta_{j}(\mathcal{B}[\alpha])-\Delta_{j}^{i}(\mathcal{B}[\alpha])\right) & \geq\left(b_{i \ldots i}-\beta_{i}(\mathcal{B})\right)\left(b_{j \ldots j}-\beta_{j}(\mathcal{B})-\Delta_{j}^{i}(\mathcal{B})\right) \\
& >\left(\beta_{j}(\mathcal{B})-b_{j i \ldots i}\right) \Delta_{i}(\mathcal{B}) \\
& \geq\left(\beta_{j}(\mathcal{B}[\alpha])-b_{j i \ldots i}\right) \Delta_{i}(\mathcal{B}[\alpha])
\end{aligned}
$$

Thus $\mathcal{A}[\alpha]$ is a quasi-double $\bar{B}$-tensor.

Definition 3.4. Let $\mathcal{A}$ be an $m$-order $n$-dimensional tensor. Then $\mathcal{A}^{+}=\left(a_{i_{1} \ldots i_{m}}^{+}\right)$is an $m$-order $n$-dimensional tensor defined as $a_{i_{1} \ldots i_{m}}^{+}=a_{i_{1} \ldots i_{m}}-\beta_{i_{1}}(\mathcal{A})$.

It is easy to see that $\mathcal{A}^{+}$is a $Z$-tensor.

Theorem 3.4. Let $\mathcal{A}$ be an m-order $n$-dimensional tensor. Then

(a) $\mathcal{A}$ is a double $\bar{B}$-tensor if and only if $\overline{\mathcal{A}}^{+}$is a double B-tensor.

(b) $\mathcal{A}$ is a quasi-double $\bar{B}$-tensor if and only if $\overline{\mathcal{A}}^{+}$is a quasi-double B-tensor.

Proof. Let $\mathcal{B}=\overline{\mathcal{A}}$ and $\mathcal{C}=\overline{\mathcal{A}}^{+}$.

(a) Suppose $\mathcal{A}$ is a double $\bar{B}$-tensor. Then $c_{i \ldots i}=b_{i \ldots i}-\beta_{i}(\mathcal{B})>0$. Since $\mathcal{C}$ is a $Z$-tensor and $\Delta_{i}(\mathcal{B})=\Delta_{i}(\mathcal{C})$, we have $c_{i \ldots i} \geq \Delta_{i}(\mathcal{C})$. Now, we have

$$
\left(c_{i \ldots i}-\beta_{i}(\mathcal{C})\right)\left(c_{j \ldots j}-\beta_{j}(\mathcal{C})\right)=\left(b_{i \ldots i}-\beta_{i}(\mathcal{B})\right)\left(b_{j \ldots j}-\beta_{j}(\mathcal{B})\right)
$$


and

$$
\Delta_{i}(\mathcal{B}) \Delta_{j}(\mathcal{B})=\Delta_{i}(\mathcal{C}) \Delta_{j}(\mathcal{C})
$$

Thus

$$
\left(c_{i \ldots i}-\beta_{i}(\mathcal{C})\right)\left(c_{j \ldots j}-\beta_{j}(\mathcal{C})\right)>\Delta_{i}(\mathcal{C}) \Delta_{j}(\mathcal{C})
$$

if and only if

$$
\left(b_{i \ldots i}-\beta_{i}(\mathcal{B})\right)\left(b_{j \ldots j}-\beta_{j}(\mathcal{B})\right)>\Delta_{i}(\mathcal{B}) \Delta_{j}(\mathcal{B}) .
$$

Hence $\mathcal{A}$ is a double $\bar{B}$-tensor if and only if $\overline{\mathcal{A}}^{+}$is a double $B$-tensor.

(b) We have

$$
\left(c_{i \ldots i}-\beta_{i}(\mathcal{C})\right)\left(c_{j \ldots j}-\beta_{j}(\mathcal{C})-\Delta_{j}^{i}(\mathcal{C})\right)=\left(b_{i \ldots i}-\beta_{i}(\mathcal{B})\right)\left(b_{j \ldots j}-\beta_{j}(\mathcal{B})-\Delta_{j}^{i}(\mathcal{B})\right)
$$

and

$$
\left(\beta_{j}(\mathcal{C})-c_{j i \ldots i}\right) \Delta_{j}^{i}(\mathcal{C})=\left(\beta_{j}(\mathcal{B})-b_{j i \ldots i}\right) \Delta_{j}^{i}(\mathcal{B})
$$

Thus

$$
\left(c_{i \ldots i}-\beta_{i}(\mathcal{C})\right)\left(c_{j \ldots j}-\beta_{j}(\mathcal{C})-\Delta_{j}^{i}(\mathcal{C})\right)>\left(\beta_{j}(\mathcal{C})-c_{j i \ldots i}\right) \Delta_{j}^{i}(\mathcal{C})
$$

if and only if

$$
\left(b_{i \ldots i}-\beta_{i}(\mathcal{B})\right)\left(b_{j \ldots j}-\beta_{j}(\mathcal{B})-\Delta_{j}^{i}(\mathcal{B})\right)>\left(\beta_{j}(\mathcal{B})-b_{j i \ldots i}\right) \Delta_{j}^{i}(\mathcal{B}) .
$$

Hence $\mathcal{A}$ is a quasi-double $\bar{B}$-tensor if and only if $\overline{\mathcal{A}}^{+}$is a quasi-double $B$-tensor.

Define

$$
\alpha_{i}(\mathcal{A})= \begin{cases}\beta_{i}(\mathcal{A}) & \text { if } a_{i \ldots i}>0 \\ \gamma_{i}(\mathcal{A}) & \text { if } a_{i \ldots i}<0\end{cases}
$$

where $\beta_{i}(\mathcal{A})$ and $\gamma_{i}(\mathcal{A})$ are defined in (6) and (17), respectively.

The following theorem gives an equivalent condition for a tensor to be a double $\bar{B}$-tensor.

Theorem 3.5. Let $\mathcal{A}$ be an m-order n-dimensional tensor. Then, $\mathcal{A}$ is a double $\bar{B}$-tensor if and only if

(a) $\left|a_{i \ldots i}\right|>\left|\alpha_{i}(\mathcal{A})\right|$ for all $i$,

(b) $\left|a_{i \ldots i}-\alpha_{i}(\mathcal{A})\right| \geq \sum_{\delta_{i i_{2} \ldots i_{m}=0}}\left|\alpha_{i}(\mathcal{A})-a_{i i_{2} \ldots i_{m}}\right|$,

(c) $\left|a_{i \ldots i}-\alpha_{i}(\mathcal{A})\right|\left|a_{j \ldots j}-\alpha_{j}(\mathcal{A})\right|>\left(\sum_{\delta_{i i_{2} \ldots i_{m}=0}}\left|\alpha_{i}(\mathcal{A})-a_{i i_{2} \ldots i_{m}}\right|\right)\left(\sum_{\delta_{j i_{2} \ldots i_{m}=0}}\left|\alpha_{j}(\mathcal{A})-a_{j i_{2} \ldots i_{m}}\right|\right)$.

Proof. Let $\mathcal{B}=\overline{\mathcal{A}}$. Then $b_{i \ldots i}>\beta_{i}(\mathcal{B})$ if and only if $a_{i \ldots i}>\beta_{i}(\mathcal{A})$ if $a_{i \ldots i}>0$ and $-a_{i \ldots i}>$ $-\gamma_{i}(\mathcal{A})$ if $a_{i \ldots i}<0$. Thus $b_{i \ldots i}>\beta_{i}(\mathcal{B})$ if and only if $\left|a_{i \ldots i}\right|>\left|\alpha_{i}(\mathcal{A})\right|$ for all $i$.

If $a_{i \ldots i}<0$, then

$$
-a_{i \ldots i}+\alpha_{i}(\mathcal{A})=b_{i \ldots i}-\beta_{i}(\mathcal{B}) \geq \sum_{\delta_{i i_{2} \ldots i_{m}}=0}\left(\beta_{i}(\mathcal{B})-b_{i i_{2} \ldots i_{m}}\right)=\sum_{\delta_{i i_{2} \ldots i_{m}}=0}\left|\alpha_{i}(\mathcal{A})-a_{i i_{2} \ldots i_{m}}\right|
$$


and if $a_{i \ldots i}>0$, then

$$
a_{i \ldots i}-\alpha_{i}(\mathcal{A})=b_{i \ldots i}-\beta_{i}(\mathcal{B}) \geq \sum_{\delta_{i i_{2} \ldots i_{m}}=0}\left(\beta_{i}(\mathcal{B})-b_{i i_{2} \ldots i_{m}}\right)=\sum_{\delta_{i i_{2} \ldots i_{m}}=0}\left|\alpha_{i}(\mathcal{A})-a_{i i_{2} \ldots i_{m}}\right| .
$$

Thus $\left|a_{i \ldots i}-\alpha_{i}(\mathcal{A})\right| \geq \sum_{\delta_{i i_{2} \ldots i_{m}=0}}\left|\alpha_{i}(\mathcal{A})-a_{i i_{2} \ldots i_{m}}\right|$ if and only if

$$
b_{i \ldots i}-\beta_{i}(\mathcal{B}) \geq \sum_{\delta_{i i_{2} \ldots i_{m}}=0}\left(\beta_{i}(\mathcal{B})-b_{i i_{2} \ldots i_{m}}\right) .
$$

Since

$$
\left|a_{i \ldots i}-\alpha_{i}(\mathcal{A})\right|\left|a_{j \ldots j}-\alpha_{j}(\mathcal{A})\right|=\left(b_{i \ldots i}-\beta_{i}(\mathcal{B})\right)\left(b_{j \ldots j}-\beta_{j}(\mathcal{B})\right)
$$

and

$$
\begin{aligned}
& \left(\sum_{\delta_{i i_{2} \ldots i_{m}}=0}\left(\beta_{i}(\mathcal{B})-b_{i i_{2} \ldots i_{m}}\right)\right)\left(\sum_{\delta_{j i_{2} \ldots i_{m}}=0}\left(\beta_{j}(\mathcal{B})-b_{j i_{2} \ldots i_{m}}\right)\right) \\
& =\left(\sum_{\delta_{i i_{2} \ldots i_{m}}=0}\left|\alpha_{i}(\mathcal{A})-a_{i i_{2} \ldots i_{m}}\right|\right)\left(\sum_{\delta_{j i_{2} \ldots i_{m}}=0}\left|\alpha_{j}(\mathcal{A})-a_{j i_{2} \ldots i_{m}}\right|\right),
\end{aligned}
$$

we have

$$
\left|a_{i \ldots i}-\alpha_{i}(\mathcal{A})\right|\left|a_{j \ldots j}-\alpha_{j}(\mathcal{A})\right|>\left(\sum_{\delta_{i i_{2} \ldots i_{m}=0}}\left|\alpha_{i}(\mathcal{A})-a_{i i_{2} \ldots i_{m}}\right|\right)\left(\sum_{\delta_{j i_{2} \ldots i_{m}=0}}\left|\alpha_{j}(\mathcal{A})-a_{j i_{2} \ldots i_{m}}\right|\right)
$$

if and only if

$$
\left(b_{i \ldots i}-\beta_{i}(\mathcal{B})\right)\left(b_{j \ldots j}-\beta_{j}(\mathcal{B})\right)>\left(\sum_{\delta_{i i_{2} \ldots i_{m}}=0}\left(\beta_{i}(\mathcal{B})-b_{i i_{2} \ldots i_{m}}\right)\right)\left(\sum_{\delta_{j i_{2} \ldots i_{m}}=0}\left(\beta_{j}(\mathcal{B})-b_{j i_{2} \ldots i_{m}}\right)\right) .
$$

This proves the result.

The following result gives an equivalent condition for a tensor to be a quasi-double $\bar{B}$ tensor.

Theorem 3.6. Let $\mathcal{A}=\left(a_{i_{1} \ldots i_{m}}\right)$ be an m-order $n$-dimensional tensor. Then $\mathcal{A}$ is a quasidouble $\bar{B}$-tensor if and only if for each $i \in[n],\left|a_{i \ldots i}\right|>\left|\alpha_{i}(\mathcal{A})\right|$ and for any $i \neq j \in[n]$,

$$
\begin{aligned}
& \left|a_{i \ldots i}-\alpha_{i}(\mathcal{A})\right|\left(\left|a_{j \ldots j}-\alpha_{j}(\mathcal{A})\right|-\sum_{\substack{\delta_{i j_{2} \ldots j_{m}=0} \\
\delta_{j_{2} \ldots j_{m}=0}}}\left|\alpha_{j}(\mathcal{A})-a_{j j_{2} \ldots j_{m}}\right|\right) \\
& >\left|\alpha_{j}(\mathcal{A})-a_{j i \ldots i}\right| \sum_{\delta_{i i_{2} \ldots i_{m}=0}}\left|\alpha_{i}(\mathcal{A})-a_{i i_{2} \ldots i_{m}}\right| .
\end{aligned}
$$


Proof. Let $\mathcal{B}=\overline{\mathcal{A}}$. By the definition of $\alpha_{i}(\mathcal{A})$, one has that $b_{i \ldots i}>\beta_{i}(\mathcal{A})$ if and only if $a_{i \ldots i}>\beta_{i}(\mathcal{A})$ if $a_{i \ldots i}>0$ and $a_{i \ldots i}<\gamma_{i}(\mathcal{A})$ if $a_{i \ldots i}<0$. Thus, we have $b_{i \ldots i}>\beta_{i}(\mathcal{A})$ if and only if $\left|a_{i \ldots i}\right|>\left|\alpha_{i}(\mathcal{A})\right|$.

Since,

$$
\begin{aligned}
& \left|a_{i \ldots i}-\alpha_{i}(\mathcal{A})\right|\left(\left|a_{j \ldots j}-\alpha_{j}(\mathcal{A})\right|-\sum_{\substack{\delta_{i j} \ldots j_{m}=0 \\
\delta_{j_{j}} \ldots j_{m}=0}}\left|\alpha_{j}(\mathcal{A})-a_{j j_{2} \ldots j_{m}}\right|\right) \\
& =\left(b_{i \ldots i}-\beta_{i}(\mathcal{B})\left(b_{j \ldots j}-\beta_{j}(\mathcal{B})-\sum_{\substack{\delta_{i j_{2} \ldots j_{m}=0} \\
\delta_{j_{2} \ldots j_{m}=0}}}\left(\beta_{j}(\mathcal{B})-b_{j j_{2} \ldots j_{m}}\right)\right.\right.
\end{aligned}
$$

if and only if

$$
\begin{aligned}
& \left(\beta_{j}(\mathcal{B})-b_{j i \ldots i}\right) \sum_{\delta_{i i_{2} \ldots i_{m}=0}}\left(\beta_{i}(\mathcal{B})-b_{i i_{2} \ldots i_{m}}\right) \\
& =\left|\alpha_{j}(\mathcal{A})-a_{j i \ldots i}\right| \sum_{\delta_{i i_{2} \ldots i_{m}=0}}\left|\alpha_{i}(\mathcal{A})-a_{i i_{2} \ldots i_{m}}\right|
\end{aligned}
$$

Thus, we have

$$
\begin{aligned}
& \left|a_{i \ldots i}-\alpha_{i}(\mathcal{A})\right|\left(\left|a_{j \ldots j}-\alpha_{j}(\mathcal{A})\right|-\sum_{\substack{\delta_{i j_{2} \ldots j_{m}=0} \\
\delta_{j j_{2} \ldots j_{m}=0}}}\left|\alpha_{j}(\mathcal{A})-a_{j j_{2} \ldots j_{m}}\right|\right) \\
& >\left|\alpha_{j}(\mathcal{A})-a_{j i \ldots i}\right| \sum_{\delta_{i i_{2} \ldots i_{m}=0}}\left|\alpha_{i}(\mathcal{A})-a_{i i_{2} \ldots i_{m}}\right| .
\end{aligned}
$$

if and only if

$$
\begin{aligned}
& \left(b_{i \ldots i}-\beta_{i}(\mathcal{B})\left(b_{j \ldots j}-\beta_{j}(\mathcal{B})-\sum_{\substack{\delta_{i j_{2} \ldots j_{m}=0} \\
\delta_{j j_{2} \ldots j_{m}=0}}}\left(\beta_{j}(\mathcal{B})-b_{j j_{2} \ldots j_{m}}\right)\right.\right. \\
& >\left(\beta_{j}(\mathcal{B})-b_{j i \ldots i}\right) \sum_{\delta_{i i_{2} \ldots i_{m}=0}}\left(\beta_{i}(\mathcal{B})-b_{i i_{2} \ldots i_{m}}\right) .
\end{aligned}
$$

This proves the result.

\section{New bounds for $H$-eigenvalues of even order sym- metric tensors}

In this section, we discuss results about location of the $H$-eigenvalues of even order symmetric tensors. First we state the following useful lemma. Proof is easy to verify.

Lemma 4.1. Let $\mathcal{A}=\left(a_{i_{1} \ldots i_{m}}\right) \in \mathcal{T}\left(\mathbb{R}^{n}, m\right)$. Then 0 is an $H$-eigenvalue of $\mathcal{A}$ if and only if 0 is an $H$-eigenvalue of $\overline{\mathcal{A}}$. 
In the following theorem we give a new region for the $H$-eigenvalues of even order symmetric tensors.

Theorem 4.1. Let $\mathcal{A}=\left(a_{i_{1} \ldots i_{m}}\right) \in \mathcal{T}\left(\mathbb{R}^{n}, m\right)$ be an even order symmetric tensor and $\lambda$ be an $H$-eigenvalue of $\mathcal{A}$. For any $i \in[n]$, Define

$$
\begin{gathered}
\Lambda_{i}=\left[a_{i \ldots i}-\beta_{i}(\mathcal{A}), a_{i \ldots i}-\gamma_{i}(\mathcal{A})\right] \\
\widetilde{\Lambda}_{i}=\left(a_{i \ldots i}-\beta_{i}(\mathcal{A})-\Delta_{i}(\mathcal{A}), a_{i \ldots i}-\gamma_{i}(\mathcal{A})-\Theta_{i}(\mathcal{A})\right)
\end{gathered}
$$

and for any $i \neq j \in[n]$,

$$
\begin{aligned}
& \Lambda_{i j}^{1}=\left\{x \in\left(-\infty, \min \left\{a_{i \ldots i}, a_{j \ldots j}\right\}\right):\left|a_{i \ldots i}-\beta_{i}(\mathcal{A})-x\right|\left|a_{j \ldots j}-\beta_{j}(\mathcal{A})-x\right| \leq \Delta_{i}(\mathcal{A}) \Delta_{j}(\mathcal{A})\right\} \\
& \Lambda_{i j}^{2}=\left\{x \in\left(a_{i \ldots i}, a_{j \ldots j}\right):\left|a_{i \ldots i}-\gamma_{i}(\mathcal{A})-x\right|\left|a_{j \ldots j}-\beta_{j}(\mathcal{A})-x\right| \leq \Theta_{i}(\mathcal{A}) \Delta_{j}(\mathcal{A})\right\} \\
& \Lambda_{i j}^{3}=\left\{x \in\left(a_{j \ldots j}, a_{i \ldots i}\right):\left|a_{i \ldots i}-\beta_{i}(\mathcal{A})-x\right|\left|a_{j \ldots j}-\gamma_{j}(\mathcal{A})-x\right| \leq \Delta_{i}(\mathcal{A}) \Theta_{j}(\mathcal{A})\right\} \\
& \Lambda_{i j}^{4}=\left\{x \in\left(\max \left\{a_{i \ldots i}, a_{j \ldots j}\right\},+\infty\right):\left|a_{i \ldots i}-\gamma_{i}(\mathcal{A})-x\right|\left|a_{j \ldots j}-\gamma_{j}(\mathcal{A})-x\right| \leq \Theta_{i}(\mathcal{A}) \Theta_{j}(\mathcal{A})\right\}
\end{aligned}
$$

where $\beta_{i}(\mathcal{A})$ and $\gamma_{i}(\mathcal{A})$ are defined in (6) and (7) . Let

$$
\Lambda_{i j}= \begin{cases}\Lambda_{i j}^{1} \cup \Lambda_{i j}^{2} \cup \Lambda_{i j}^{4}, & \text { if } a_{i \ldots i} \leq a_{j \ldots j}, \\ \Lambda_{i j}^{1} \cup \Lambda_{i j}^{3} \cup \Lambda_{i j}^{4}, & \text { if } a_{i \ldots i} \geq a_{j \ldots j} .\end{cases}
$$

Then,

$$
\lambda \in \Lambda(\mathcal{A})=\left(\bigcup_{i=1}^{n} \Lambda_{i}\right) \cup\left(\bigcup_{i=1}^{n} \widetilde{\Lambda}_{i}\right) \cup\left(\bigcup_{\substack{i, j=1 \\ i \neq j}}^{n} \Lambda_{i j}\right) .
$$

Proof. Let $\lambda$ be an $H$-eigenvalue of $\mathcal{A}$. Suppose that $\lambda \notin \Lambda(\mathcal{A})$. Observe that $\mathcal{A}-\lambda \mathcal{I}$ and $\mathcal{A}$ have the same off-diagonal elements. Since $\lambda \notin \Lambda_{i}$, we have either $a_{i \ldots i}-\lambda>\beta_{i}(\mathcal{A})$ or $a_{i \ldots i}-\lambda<\gamma_{i}(\mathcal{A})$ for all $i \in[n]$. Thus $\left|a_{i \ldots i}-\lambda\right|>\left|\alpha_{i}(\mathcal{A})\right|=\left|\alpha_{i}(\mathcal{A}-\lambda \mathcal{I})\right|$ for all $i \in[n]$. Since $\lambda \notin \widetilde{\Lambda}_{i}$, we have either $\left(a_{i \ldots i}-\beta_{i}(\mathcal{A})-\Delta_{i}(\mathcal{A})-\lambda\right) \geq 0$ or $\left(a_{i \ldots i}-\gamma_{i}(\mathcal{A})-\Theta_{i}(\mathcal{A})-\lambda\right) \leq 0$. Thus $\left|a_{i \ldots i}-\lambda-\alpha_{i}(\mathcal{A}-\lambda \mathcal{I})\right| \geq \sum_{\delta_{i i_{2} \ldots i_{m}=0}}\left|\alpha_{i}(\mathcal{A}-\lambda \mathcal{I})-a_{i i_{2} \ldots i_{m}}\right|$.

Let $i, j \in[n]$ and $i \neq j$. We may assume without loss of generality that $a_{i \ldots i} \leq a_{j \ldots j}$. If $\lambda \in\left(-\infty, a_{i \ldots i}\right)$, then $a_{i \ldots i}-\lambda>0, a_{j \ldots j}-\lambda>0$ and

$$
\left|a_{i \ldots i}-\beta_{i}(\mathcal{A})-\lambda\right|\left|a_{j \ldots j}-\beta_{j}(\mathcal{A})-\lambda\right|>\Delta_{i}(\mathcal{A}) \Delta_{j}(\mathcal{A}) .
$$

If $\lambda \in\left(a_{i \ldots i}, a_{j \ldots j}\right)$, then $a_{i \ldots i}-\lambda<0, a_{j \ldots j}-\lambda>0$ and

$$
\left|a_{i \ldots i}-\gamma_{i}(\mathcal{A})-\lambda\right|\left|a_{j \ldots j}-\beta_{j}(\mathcal{A})-\lambda\right|>\Theta_{i}(\mathcal{A}) \Delta_{j}(\mathcal{A})
$$


If $\lambda \in\left(a_{j \ldots j}, \infty\right)$, then $a_{i \ldots i}-\lambda<0, a_{j \ldots j}-\lambda<0$ and

$$
\left|a_{i \ldots i}-\gamma_{i}(\mathcal{A})-\lambda\right|\left|a_{j \ldots j}-\gamma_{j}(\mathcal{A})-\lambda\right|>\Theta_{i}(\mathcal{A}) \Theta_{j}(\mathcal{A})
$$

From the above observations, we have the following inequality

$$
\begin{aligned}
& \left|a_{i \ldots i}-\lambda-\alpha_{i}(\mathcal{A}-\lambda \mathcal{I})\right|\left|a_{j \ldots j}-\lambda-\alpha_{j}(\mathcal{A}-\lambda \mathcal{I})\right| \\
& \quad>\left(\sum_{\delta_{i i_{2} \ldots i_{m}=0}}\left|\alpha_{i}(\mathcal{A}-\lambda \mathcal{I})-a_{i i_{2} \ldots i_{m}}\right|\right)\left(\sum_{\delta_{j i_{2} \ldots i_{m}=0}}\left|\alpha_{j}(\mathcal{A}-\lambda \mathcal{I})-a_{j i_{2} \ldots i_{m}}\right|\right) .
\end{aligned}
$$

Thus, by Theorem 3.5, we have $\mathcal{A}-\lambda \mathcal{I}$ is a double $\bar{B}$-tensor. By Theorem 2.1 (a) and Lemma 4.1, 0 is not an $H$-eigenvalue of $\mathcal{A}-\lambda \mathcal{I}$. Thus there does not exist a nonzero vector $x \in \mathbb{R}^{n}$ such that $(\mathcal{A}-\lambda \mathcal{I}) x^{m-1}=0$, which implies $\lambda$ is not an $H$-eigenvalue of $\mathcal{A}$. This is a contradiction. Therefore, $\lambda \in \Lambda(\mathcal{A})$.

The above theorem is an analogous version of [7, Theorem 3.3] for tensors. The following theorem is a refinement of the above theorem. The eigenvalue region we get in this theorem are smaller than the above one.

Theorem 4.2. Let $\mathcal{A}=\left(a_{i_{1} \ldots i_{m}}\right) \in \mathcal{T}\left(\mathbb{R}^{n}, m\right)$ be an even order symmetric tensor and $\lambda$ be an $H$-eigenvalue of $\mathcal{A}$. Define

$$
\Psi_{i}=\left[a_{i \ldots i}-\beta_{i}(\mathcal{A}), a_{i \ldots i}-\gamma_{i}(\mathcal{A})\right], \quad i \in[n]
$$

and for any $i \neq j \in[n]$,

$$
\begin{aligned}
& \Psi_{i j}^{1}=\left\{x \in\left(-\infty, \min \left\{a_{i \ldots i}, a_{j \ldots j}\right\}\right):\left|a_{i \ldots i}-\beta_{i}(\mathcal{A})-x\right|\left(\left|a_{j \ldots j}-\beta_{j}(\mathcal{A})-x\right|-\Delta_{j}^{i}(\mathcal{A})\right)\right. \\
&\left.\leq\left(\beta_{j}(\mathcal{A})-a_{j i \ldots i}\right) \Delta_{i}(\mathcal{A})\right\}, \\
& \Psi_{i j}^{2}=\left\{x \in\left(a_{i \ldots i}, a_{j \ldots j}\right):\left|a_{i \ldots i}-\gamma_{i}(\mathcal{A})-x\right|\left(\left|a_{j \ldots j}-\beta_{j}(\mathcal{A})-x\right|-\Delta_{j}^{i}(\mathcal{A})\right) \leq\left(\beta_{j}(\mathcal{A})-a_{j i \ldots i}\right) \Theta_{i}(\mathcal{A})\right\}, \\
& \Psi_{i j}^{3}=\left\{x \in\left(a_{j \ldots j}, a_{i \ldots i}\right):\left|a_{i \ldots i}-\beta_{i}(\mathcal{A})-x\right|\left(\left|a_{j \ldots j}-\gamma_{j}(\mathcal{A})-x\right|-\Theta_{j}^{i}(\mathcal{A})\right) \leq\left(a_{j i \ldots i}-\gamma_{j}(\mathcal{A})\right) \Delta_{i}(\mathcal{A})\right\}, \\
& \\
& \Psi_{i j}^{4}=\left\{x \in\left(\max \left\{a_{i \ldots i}, a_{j \ldots j}\right\},+\infty\right):\left|a_{i \ldots i}-\gamma_{i}(\mathcal{A})-x\right|\left(\left|a_{j \ldots j}-\gamma_{j}(\mathcal{A})-x\right|-\Theta_{j}^{i}(\mathcal{A})\right)\right. \\
&\left.\leq\left(a_{j i \ldots i}-\gamma_{j}(\mathcal{A})\right) \Theta_{i}(\mathcal{A})\right\},
\end{aligned}
$$

where $\beta_{i}(\mathcal{A})$ and $\gamma_{i}(\mathcal{A})$ are defined in (6) and (7). Let

$$
\Psi_{i j}= \begin{cases}\Psi_{i j}^{1} \cup \Psi_{i j}^{2} \cup \Psi_{i j}^{4}, & \text { if } a_{i \ldots i} \leq a_{j \ldots j} \\ \Psi_{i j}^{1} \cup \Psi_{i j}^{3} \cup \Psi_{i j}^{4}, & \text { if } a_{i \ldots i} \geq a_{j \ldots j}\end{cases}
$$

Then,

$$
\lambda \in \Psi(\mathcal{A})=\left(\bigcup_{i=1}^{n} \Psi_{i}\right) \cup\left(\bigcup_{\substack{i, j=1 \\ i \neq j}}^{n} \Psi_{i j}\right) .
$$


Proof. Let $\lambda$ be an $H$-eigenvalue of $\mathcal{A}$. Suppose that $\lambda \notin \Psi(\mathcal{A})$. Then for any $i \in[n]$, $\left|a_{i \ldots i}-\lambda\right|>\left|\alpha_{i}(\mathcal{A}-\lambda \mathcal{I})\right|$. Let $i \neq j \in[n]$. Without loss of generality, assume $a_{i \ldots i} \leq a_{j \ldots j}$. If $\lambda \in\left(-\infty, a_{i \ldots i}\right)$, then

$$
\left|a_{i \ldots i}-\beta_{i}(\mathcal{A})-\lambda\right|\left(\left|a_{j \ldots j}-\beta_{j}(\mathcal{A})-\lambda\right|-\Delta_{j}^{i}(\mathcal{A})\right)>\left(\beta_{j}(\mathcal{A})-a_{j i \ldots i}\right) \Delta_{i}(\mathcal{A}) .
$$

If $\lambda \in\left(a_{i \ldots . i}, a_{j \ldots j}\right)$, then

$$
\left|a_{i \ldots i}-\gamma_{i}(\mathcal{A})-\lambda\right|\left(\left|a_{j \ldots j}-\beta_{j}(\mathcal{A})-\lambda\right|-\Delta_{j}^{i}(\mathcal{A})\right)>\left(\beta_{j}(\mathcal{A})-a_{j i \ldots i}\right) \Theta_{i}(\mathcal{A}) .
$$

If $\lambda \in\left(a_{j \ldots j}, \infty\right)$, then

$$
\left|a_{i \ldots i}-\gamma_{i}(\mathcal{A})-\lambda\right|\left(\left|a_{j \ldots j}-\gamma_{j}(\mathcal{A})-\lambda\right|-\Theta_{j}^{i}(\mathcal{A})>\left(a_{j i \ldots i}-\gamma_{j}(\mathcal{A})\right) \Theta_{i}(\mathcal{A}) .\right.
$$

Thus in all three cases we have,

$$
\begin{aligned}
& \left|a_{i \ldots i}-\lambda-\alpha_{i}(\mathcal{A}-\lambda \mathcal{I})\right|\left(\left|a_{j \ldots j}-\lambda-\alpha_{j}(\mathcal{A}-\lambda \mathcal{I})\right|-\sum_{\substack{\delta_{i j_{2} \ldots j_{m}=0} \\
\delta_{j j_{2} \ldots j_{m}=0}}}\left|\alpha_{j}(\mathcal{A}-\lambda \mathcal{I})-a_{j j_{2} \ldots j_{m}}\right|\right) \\
& >\left|\alpha_{j}(\mathcal{A}-\lambda \mathcal{I})-a_{j i \ldots i}\right| \sum_{\delta_{i i_{2} \ldots i_{m}=0}}\left|\alpha_{i}(\mathcal{A}-\lambda \mathcal{I})-a_{i i_{2} \ldots i_{m}}\right| .
\end{aligned}
$$

By Theorem 3.6, we have $\mathcal{A}-\lambda \mathcal{I}$ is a quasi-double $\bar{B}$-tensor. Also, by Theorem 2.1 (b) and Lemma 4.1, 0 is not an $H$-eigenvalue of $\mathcal{A}-\lambda \mathcal{I}$. Thus there does not exit a nonzero vector $x \in \mathbb{R}^{n}$ such that $(\mathcal{A}-\lambda \mathcal{I}) x^{m-1}=0$, which means $\lambda$ is not an $H$-eigenvalue of $\mathcal{A}$. This is a contradiction. Therefore, $\lambda \in \Psi(\mathcal{A})$.

We call $\Lambda(\mathcal{A})$ and $\Psi(\mathcal{A})$ the double $\bar{B}$-intervals and quasi-double $\bar{B}$-intervals of $\mathcal{A}$, respectively. Observe that the double $\bar{B}$-intervals of $\mathcal{A}$ and quasi-double $\bar{B}$-intervals have a nature similar to the Brauer-type eigenvalues inclusion set $\Omega(\mathcal{A})$ stated in Theorem 1.3. Now, a question is nature, when range is better for determining the location of the $H$-eigenvalues of $\mathcal{A}$. We will discuss this interesting question in the following section.

\section{Comparisons between Brauer-type eigenvalues in- clusion set, double $\bar{B}$-intervals and quasi-double $\bar{B}$ - intervals}

We begin this section with the following observation. Li et al., [3, Proposition 4] proved that if $\mathcal{A}$ is a double $B$-tensor, then $\mathcal{A}$ is a quasi-double $B$-tensor. Thus we have, if $\mathcal{A}$ is a double $\bar{B}$-tensor, then $\mathcal{A}$ is a quasi-double $\bar{B}$-tensor. In the next theorem, we prove the quasi-double $\bar{B}$-intervals is contained in the double $\bar{B}$-intervals.

Theorem 5.1. Let $\mathcal{A}=\left(a_{i_{1} \ldots i_{m}}\right) \in \mathcal{T}\left(\mathbb{R}^{n}, m\right)$ be an even order symmetric tensor and $\lambda$ be an $H$-eigenvalue of $\mathcal{A}$. Then,

$$
\Psi(\mathcal{A}) \subseteq \Lambda(\mathcal{A})
$$

where $\Lambda(\mathcal{A})$ and $\Psi(\mathcal{A})$ are defined in (17) and (18), respectively. 
Proof. Let $\lambda \notin \Lambda(\mathcal{A})$. Then, by Theorem [3.5, $\mathcal{A}-\lambda \mathcal{I}$ is a double $\bar{B}$-tensor. By the above observation, $\mathcal{A}-\lambda \mathcal{I}$ is a quasi-double $\bar{B}$-tensor. Thus, by Theorem $[3.6$, we have $\lambda \notin \Psi(\mathcal{A})$. Hence $\Psi(\mathcal{A}) \subseteq \Lambda(\mathcal{A})$.

The following example shows that $\Psi(\mathcal{A})$ and Brauer-type eigenvalues inclusion set are not comparable.

Example 5.1. Consider the symmetric tensors $\mathcal{A}_{1}=\left(a_{i_{1} i_{2} i_{3} i_{4}}\right)$ and $\mathcal{A}_{2}=\left(b_{i_{1} i_{2} i_{3} i_{4}}\right)$ of order 4 dimension 2 defined as follows:

$$
\begin{aligned}
& a_{1111}=18, \quad a_{2222}=20, \quad a_{1222}=a_{2122}=a_{2212}=a_{2221}=3, \\
& a_{1122}=a_{2211}=a_{1221}=a_{2112}=a_{2121}=a_{1212}=2, \\
& a_{1112}=a_{2111}=a_{1211}=a_{1121}=2,
\end{aligned}
$$

and

$$
\begin{aligned}
& b_{1111}=2, \quad b_{2222}=6, \quad b_{1222}=b_{2122}=b_{2212}=b_{2221}=4, \\
& b_{1122}=b_{2211}=b_{1221}=b_{2112}=b_{2121}=b_{1212}=-2, \\
& b_{1112}=b_{2111}=b_{1211}=b_{1121}=5 .
\end{aligned}
$$

By using the methods of Theorem 1.3 and Theorem 4.2, one can get the location of $H$-eigenvalues of $\mathcal{A}_{1}$ and $\mathcal{A}_{2}$ listed in the following Table 1.

Table 1: Comparisons of Brauer-type eigenvalues inclusion set and quasi-double $\bar{B}$-intervals

\begin{tabular}{lll}
\hline & $\mathcal{A}_{1}$ & $\mathcal{A}_{2}$ \\
\hline Brauer-type eigenvalues inclusion set & {$[3,36.6119]$} & {$[-22.2560,28.6844]$} \\
quasi-double $\bar{B}$-intervals & {$[9,36.6119]$} & {$[-24.9257,32.6068]$} \\
H-eigenvalues & $15,35.1469$ & $-20.2289, \quad 16.0666$ \\
\hline
\end{tabular}

As we can see in the table above, the quasi-double $\bar{B}$-intervals is better when estimating the location of $H$-eigenvalues of $\mathcal{A}_{1}$ while the Brauer-type eigenvalues inclusion set is more precise for locating the $H$-eigenvalues of $\mathcal{A}_{2}$.

So, there is no indication that $\Psi(\mathcal{A})$ is tighter than $\Omega(\mathcal{A})$ or $\Omega(\mathcal{A})$ is tighter than $\Psi(\mathcal{A})$. They supplement each other.

Now, we construct a more precise region by using the results of Theorem 1.3 and Theorem 4.2 .

Theorem 5.2. Let $\mathcal{A}=\left(a_{i_{1} \ldots i_{m}}\right) \in \mathcal{T}\left(\mathbb{R}^{n}, m\right)$ be an even order symmetric tensor and $\Omega(\mathcal{A})$, $\Psi(\mathcal{A})$ be defined in (5), (18), respectively. If $\lambda$ is an $H$-eigenvalue of $\mathcal{A}$, then

$$
\lambda \in \Upsilon(\mathcal{A})=\Omega(\mathcal{A}) \cap \Psi(\mathcal{A}) .
$$




\section{Concluding remarks}

We introduced two new classes of tensors called double $\bar{B}$-tensors and quasi-double $\bar{B}$-tensors and established some of their properties. Using the properties, we derived two regions $\Lambda(\mathcal{A})$, $\Psi(\mathcal{A})$ called double $\bar{B}$-intervals, quasi-double $\bar{B}$-intervals, respectively. We observed that quasi-double $\bar{B}$-intervals are smaller than double $\bar{B}$-intervals. We discussed the relationship between Brauer-type eigenvalues inclusion set $\Omega(\mathcal{A})$ and the quasi-double $\bar{B}$-intervals $\Psi(\mathcal{A})$ and constructed the region $\Upsilon(\mathcal{A})$, which is more precise for the location of the $H$-eigenvalues of even order symmetric tensors. We investigated the location of $H$-eigenvalues of even order symmetric tensors. Do the double $\bar{B}$-intervals $\Lambda(\mathcal{A})$ and the quasi-double $\bar{B}$-intervals $\Psi(\mathcal{A})$ hold for even order nonsymmetric tensors and odd order tensors? In the future, we will research these problems.

\section{Acknowledgement}

The authors would like to thank Prof. Yaotang Li for his useful comments. The second author is supported by the German-Israeli Foundation for Scientific Research and Development (GIF) grant no. 1135-18.6/2011.

\section{References}

[1] Zhongming Chen and Liqun Qi, Circulant tensors with applications to spectral hypergraph theory and stochastic process, arXiv:1312.2752v7.

[2] Zhongming Chen, Qingzhi Yang, and Lu Ye, Further results on B-tensors with application to the location of real eigenvalues, arXiv:1408.4634.

[3] Chaoqian Li and Yaotang Li, Double B-tensors and quasi-double B-tensors, Linear Algebra Appl. 466 (2015), 343-356. MR 3278256

[4] Chaoqian Li, Yaotang Li, and $\mathrm{Xu}$ Kong, New eigenvalue inclusion sets for tensors, Numer. Linear Algebra Appl. 21 (2014), no. 1, 39-50. MR 3150608

[5] Chaoqian Li, Feng Wang, Jianxing Zhao, Yan Zhu, and Yaotang Li, Criterions for the positive definiteness of real supersymmetric tensors, J. Comput. Appl. Math. 255 (2014), 1-14.

[6] Jiawang Nie, Discriminants and nonnegative polynomials, J. Symbolic Comput. 47 (2012), no. 2, 167-191.

[7] J. M. Peña, On an alternative to Gerschgorin circles and ovals of Cassini, Numer. Math. 95 (2003), no. 2, 337-345. MR 2001081 (2004f:65045) 
[8] Liqun Qi, Eigenvalues of a real supersymmetric tensor, J. Symbolic Comput. 40 (2005), no. $6,1302-1324$.

[9] Liqun Qi and Yisheng Song, An even order symmetric B tensor is positive definite, Linear Algebra Appl. 457 (2014), 303-312. MR 3230448

[10] Jia-Yu Shao, A general product of tensors with applications, Linear Algebra Appl. 439 (2013), no. 8, 2350-2366. MR 3091308 